\title{
The Role of Ferroelectric Nanodomains in the Transport Properties of Perovskite Solar Cells Supporting Information
}

\author{
Alessandro Pecchia†, Desirée Gentilini§, Daniele Rossi§, Matthias Auf der Maur§, Aldo Di Carlo§ \\ † Consiglio Nazionale delle Ricerche, ISMN, Via Salaria km 29.300, 00017 Monterotondo, Italy \\ § Dipartimento Ing. Elettronica, Università di Roma 'Tor Vergata', Via del Politecnico 1, 00133 Roma, Italy \\ POTENTIAL PROFILE
}

The potential profile obtained for $\mathrm{P}=0.13 \mathrm{C} / \mathrm{m}^{2}$ and $\varepsilon_{r}=6.1$ is shown in Figure $\mathrm{S} .1$. In the figure, it is possible to see how nanodomains induce potential oscillations of $\pm 0.5 \mathrm{eV}$ of the band edges. The calculation has been obtained at equilibrium.
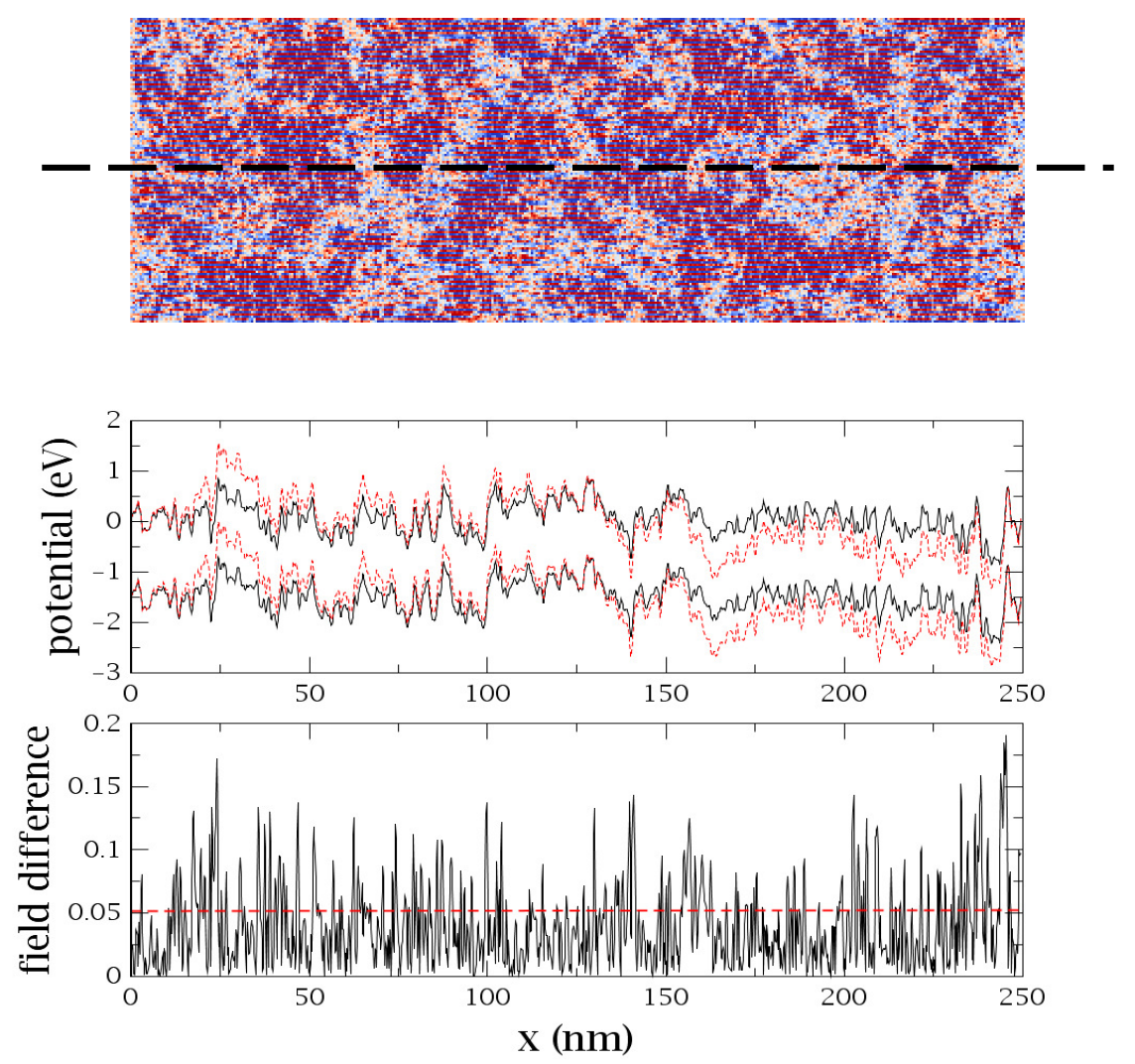

Fig. S.1 Potential profile along the shown cut-line responsible for the confinement of electrons and holes. The dotted lines is the potential due to polarizations only whereas the solid lines includes free carriers densities. The bottom panel represents the r.m.s. of the relative difference of electrostatic field. 
The two dimensional simulations shown in the text are averaged over 10 realizations starting the MMC with different random seeds. All simulations converge in the energy after $10^{8}$ iterations, depending on the polarization magnitude. The energy vs MMC steps are shown in Fig. S.2. In Figure S.3 we show the different I-V characteristics obtained for each sample. It is possible to see that $V_{o c}$ is insensitive to sample variations, whereas $J_{s c}$ varies from sample to sample. This effect depends on sample
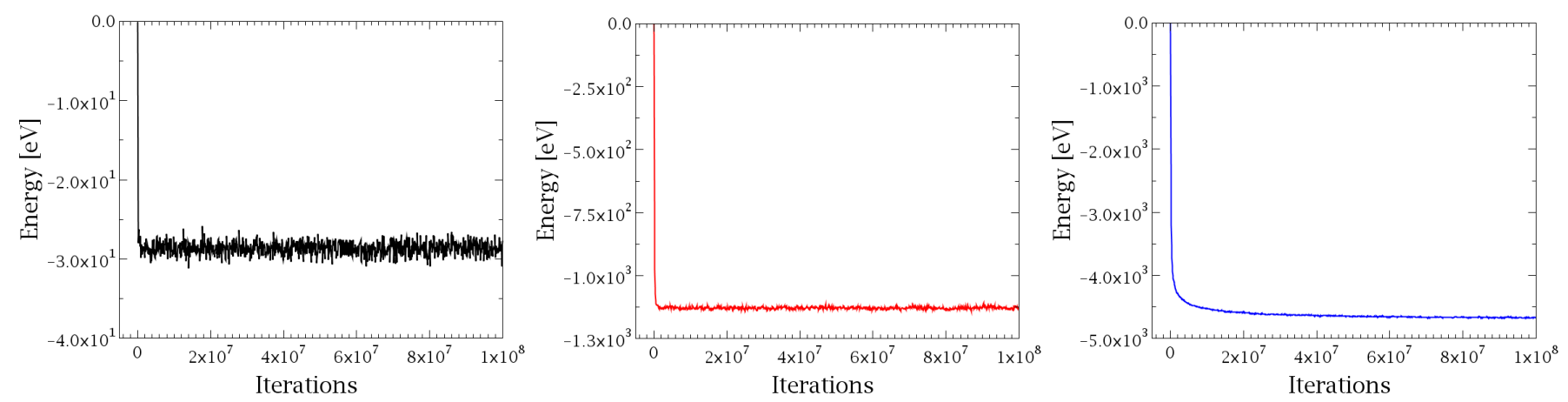

Fig. S.2 Energy vs MC step for different polarization magnitudes, from left to right for $\mathrm{P}=0.03,0.08,0.13 \mathrm{C} / \mathrm{cm}^{2}$, respectively.
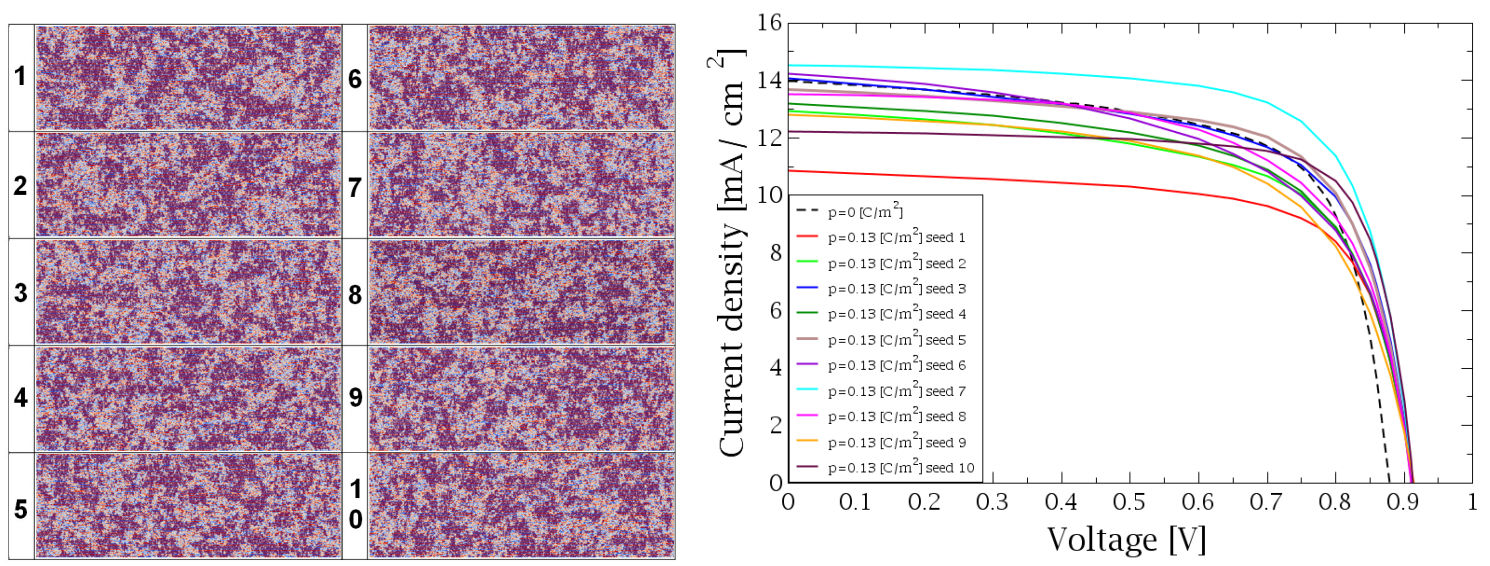

Fig. S.3 (Left) MMC Random samples starting from 10 different seeds all at $\mathrm{p}=0.13 \mathrm{C} / \mathrm{m}^{2}$ and $\mathrm{T}=300 \mathrm{~K}$. (Right) I-V characteristics for each independent sample. 
In order to validate the 2D MMC simulations, we have also performed 3D simulations on supercells of 50x50x50 with periodic boundary conditions. This is an important point since adding an extra dimension may easily suppress ordering. In such simulations, performed at $\mathrm{T}=300 \mathrm{~K}$, we have compared both the case of a uniform (spherical) distribution of orientation of the cell dipoles with the case of discrete orientations, along the cell faces. The latter is supported in references [11] and [12] of the manuscript. In both cases we find the formation of nanodomains, although in the case of discrete orientations they are more clearly visible, as in figure S.4.
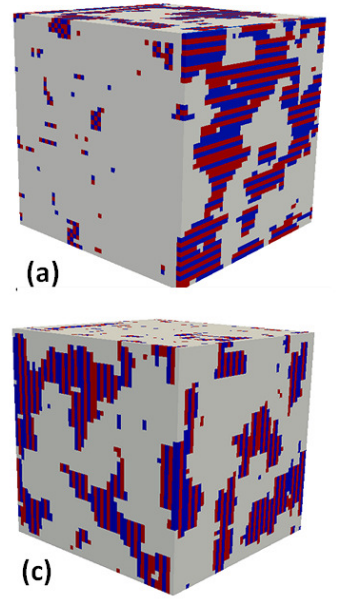
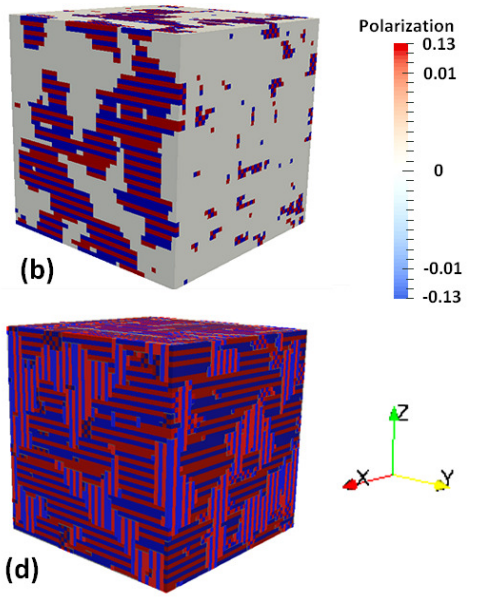

Fig. S.4 Anti-ferroelectric domain formation in a 3D supercell at $\mathrm{T}=300 \mathrm{~K}$ and for $\mathrm{P}=0.13 \mathrm{C} / \mathrm{m}^{2}$ and $\varepsilon_{r}=6.1$. Panels a-c) represent $\mathrm{P}_{\mathrm{x}}, \mathrm{P}_{\mathrm{y}}, \mathrm{P}_{\mathrm{z}}$ components of the polarization. Panel d) represents is the superposition of a,b,c.

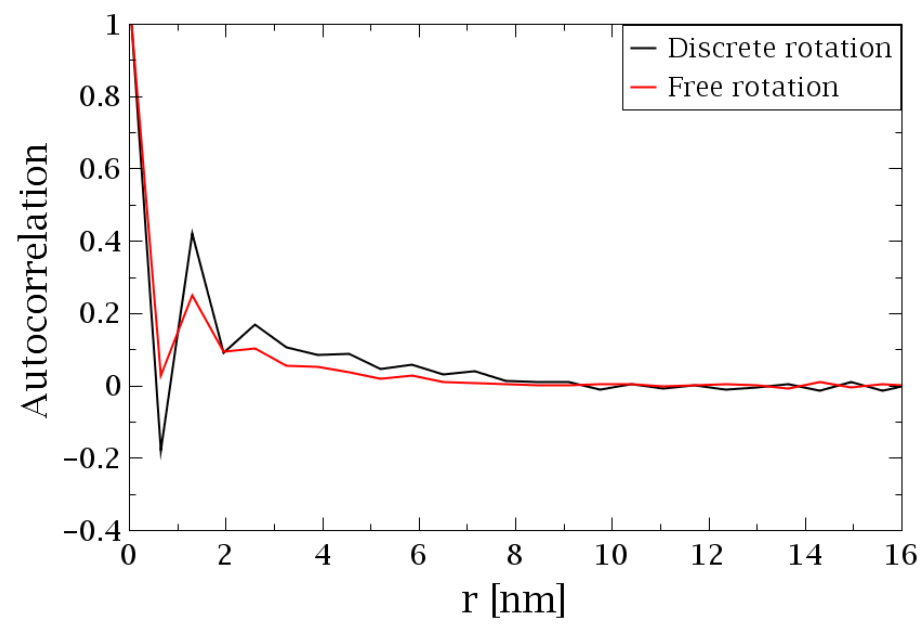

Fig. S.5 Autocorrelation function of the $\mathrm{p}_{\mathrm{x}}$. Comparisons between the discrete (black) with uniform orientations (violet).

When dipole moments are free to orient in any direction the domain are less clear. However when investigated quantitatively by mean of an autocorrelation function, then in both cases we observe a quite similar pattern. Figure S.5 compares the autocorrelation function for $p_{x}$ computed as

$A(y)=\int p_{x}\left(y^{\prime}\right) p_{x}\left(y+y^{\prime}\right) d y^{\prime}$

Both plots show the anti-ferroelectric order of neighboring cells and a dump of oscillations that goes to 0 after $8 \mathrm{~nm}$ in the case of discrete orientations and $7 \mathrm{~nm}$ in the case of random orientations. The nanodomain formation shown in the paper are consistent with these, demonstrating that the $2 \mathrm{D}$ approximation does not introduce substantial artefacts. 


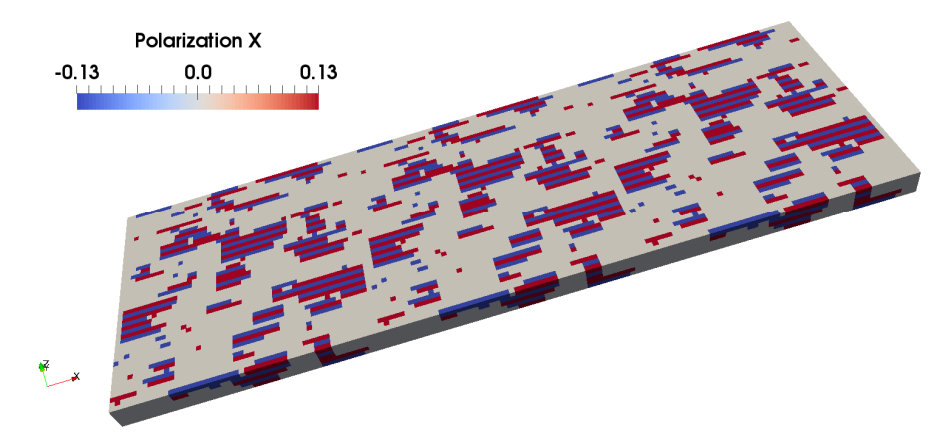

Fig. S.4 A slice of 150x50x5 cells used for the drift-diffusion simulations.

In order to assess the validity of the 2D drift-diffusion calculations, we have performed 'quasi' 3D simulations.

First we run MMC on a 150x50x50 cells. Then we performed DD simulations on a reduced model, of 150x50x5 cells, owing to computational constrains. With the mesh division used in this case of $0.32 \mathrm{~nm}$, we get around 1,500,000 number of elements, that is hitting the memory limit of our computing facility.
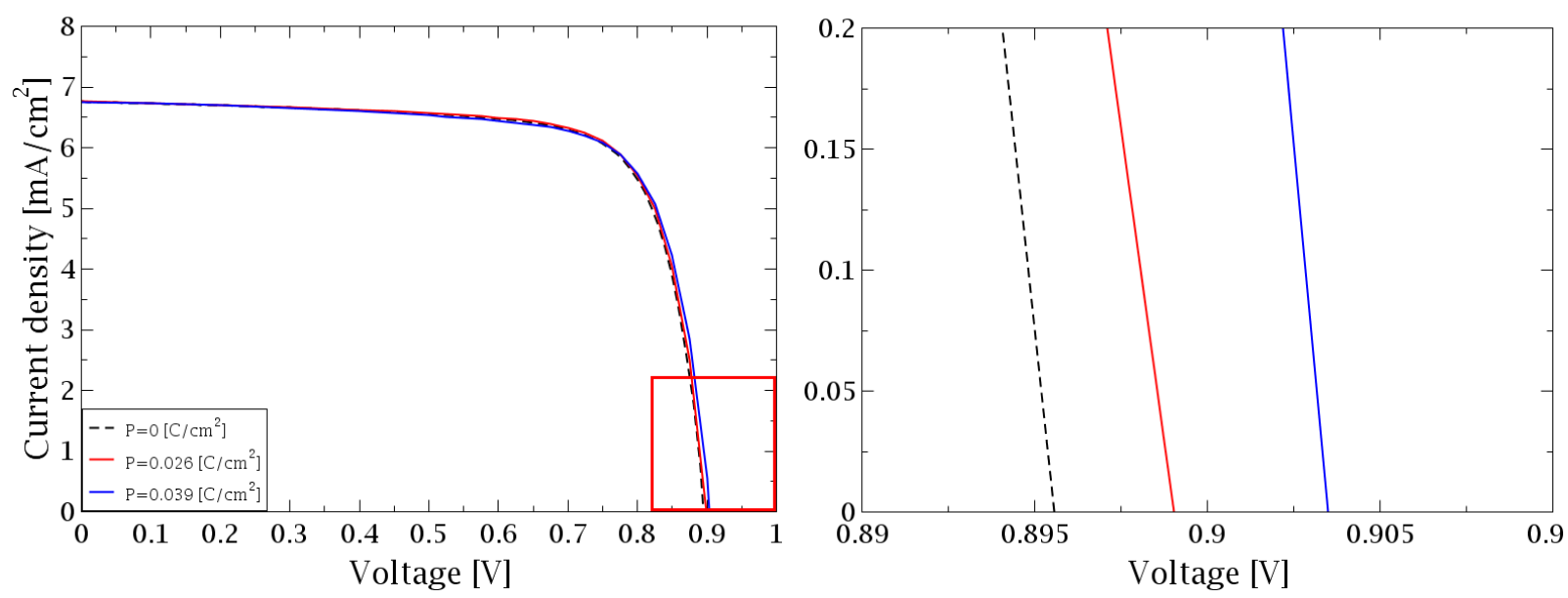

Fig. S.5 I-V characteristics of the 3D drift-diffusion simulations.

We point out that the 3D simulations are much harder to converge than the 2D counterparts. This is due to the increased amount of degree of freedom that imposes a considerable mesh coarsening (in the 2D simulations we used a mesh size of $0.15 \mathrm{~nm}$ ). In order to improve convergence of the DD equations we introduced a gradual switch-on of the polarization field, by considering only a fraction of full polarization strength $\left(\mathrm{P}=0.13 \mathrm{C} / \mathrm{cm}^{2}\right)$. Up to date we have not been able to converge the calculations beyond $30 \%$ of the polarization strength, corresponding to $\mathrm{P}=0.039 \mathrm{C} / \mathrm{cm}^{2}$. This is sufficient, though, to increase carrier localization at the domain boundaries and decrease SRH recombination. Indeed the plots of Figure S.5 confirm the findings of the 2D simulations discussed in the paper, in particular the increase of $V_{o c}$. The increase in $V_{o c}$ by 8 $\mathrm{mV}$, suggests that at the full polarization strength we may expect $\Delta V_{o c} \approx 35 \mathrm{mV}$.

The current density of the 3D simulations is much less affected by nanodomains compared to the 2D simulations shown in the paper and in Fig. S3. This is due to the fact that in 3D photocarriers can exploit the third dimension to efficiently diffuse to the electrodes, as shown in the pathways of Fig. S.6. In support of this point we find that the in the 3D simulations $I_{s c}$ slightly increases, as expected for a reduced amount of recombination. 


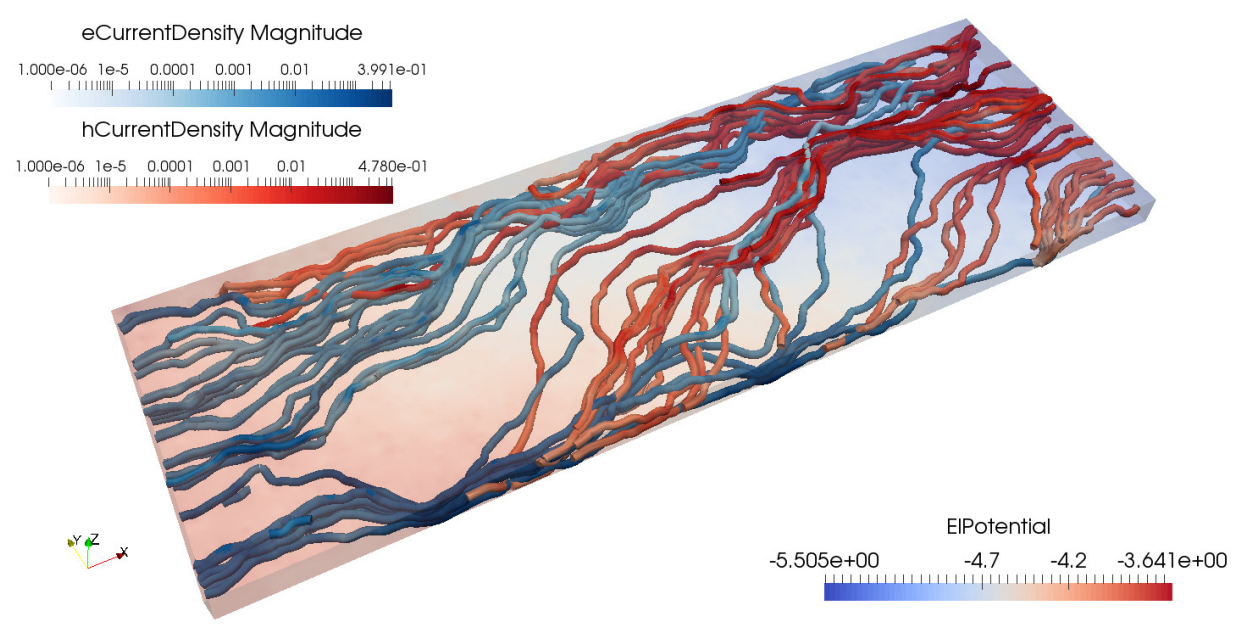

Fig. S.6 Current pathways in 3D simulations

\section{THREE-DIMENSIONAL DOMAIN EVOLUTION}

In order to compare the diffusion and molecular dipole rotation timescales we have employed our MMC to simulate the time evolution of molecular switches, restricting dipole orientations only in 6 discrete directions along the faces of the perovskite unit cells. We have considered a cubic supercell with side length $\mathrm{L}=32.5 \mathrm{~nm}$ and periodic boundary conditions. Assuming diffusive carrier motion, we estimate a transversal time of this cube of 40 ps (for a mobility of $10 \mathrm{~cm}^{2} / \mathrm{Vs}$ ). Considering that 3 ps is the average switching time of a single dipole, we wait for as many switches events such that on average every dipole has switched once. Since in the cube we have 50x50x50=125,000 cells and considering the rejection rate of $99.54 \%$ when steady state is reached, we obtain that 3 ps correspond to 27 million attempt steps of the MMC. Hence, the 45 ps of transverse time correspond to 405 million attempt steps. In Figure S.7 we report the evolution of the nanodomains on a plane $\mathrm{XY}$ cutted in the middle of the cube $(\mathrm{Z}=16.25 \mathrm{~nm})$. It is possible to see that the domain boundaries fluctuate during the typical transverse time, but evolve slowly, hence we expect that electrons and holes remain separated. On the other hand, the typical transverse time of a crystal unit cell of $0.65 \mathrm{~nm}$ is 0.016 ps which is much smaller than 3 ps. Additionally, during the switching timescale of 3 ps the carrier travels a diffusion distance of $8.8 \mathrm{~nm}$, which is larger or comparable to the domain size.
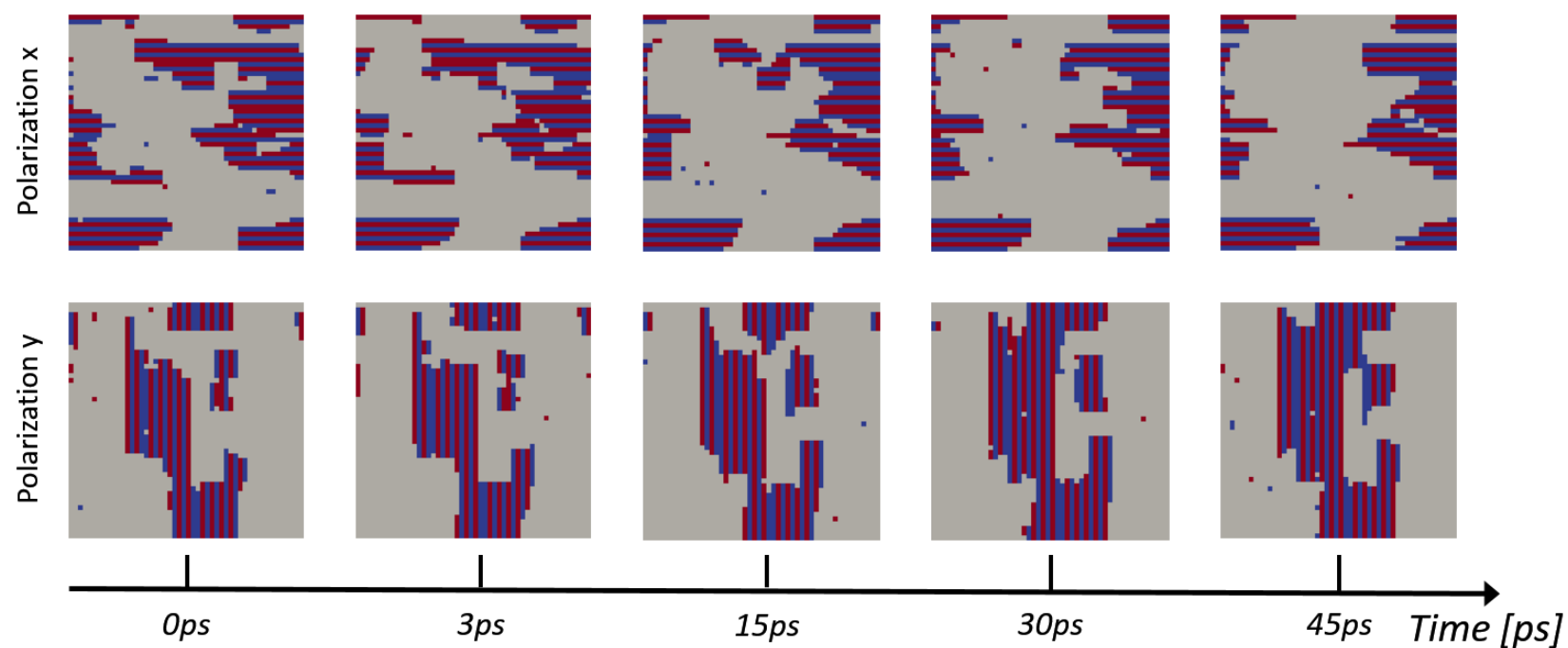

Fig. S.7 Domain variations during the MC run. MC steps have been projected to time by counting the amount of dipole rotations and knowing the average rotation timescale of 3 ps [18] 